\title{
EL PERIODISMO EN EL SIGLO DE LAS REDES SOCIALES
}

Eva Herrero-Curiel 1: Universidad Carlos III. Madrid. España eva.herrero@uc3m.es

\section{RESUMEN}

El siguiente artículo expone y analiza los cambios que las redes sociales han producido en la profesión periodística. Sistemas de producción, contenidos o modelos de negocio han sido los principales protagonistas de este cambio en el nuevo escenario interactivo, pero existe un actor importante que es el periodista. El trabajo de este profesional ha sido completamente transformado por las nuevas tecnologías. Acceso a la información, fuentes o sistemas de verificación se han visto modificados por el uso de las redes sociales como herramienta principal en su trabajo diario dentro de las redacciones. Es necesario investigar de qué manera están utilizando los periodistas estos Social Media y estudiar cuáles pueden ser las claves para que estas nuevas herramientas digitales se conviertan en un recurso útil para el periodista del siglo XXI.

PALABRAS CLAVE: Periodismo - Redes sociales - Nuevas tecnologías - Internet

\section{THE JOURNALIST IN THE SOCIAL MEDIA CENTURY}

\begin{abstract}
This paper presents and analyzes the changes produced by Social Media in the journalism profession. Systems of production, contents or models of business have been the main actors of this change in the new interactive scene, but there is an important actor: the journalist. The work of this professional has been completely transformed by the new technologies. Access to information, sources or systems of verification have been altered by the use of the Social Networks like main tool in their daily work in newsroom. We need to research how the journalists are using this Social Media in the editorial staff and study which are the keys in order that these new tools become an useful resource to the journalist of the 21st century.
\end{abstract}

\footnotetext{
${ }^{1}$ Autor correspondiente

Adolfo Carratalá: Docente e Investigador en Formación FPU. Universitat de València. Valencia, España. Correo: adolfo.carratala@uv.es
} 
KEY WORDS: Journalism - Social Media - New Technologies - Internet

\section{INTRODUCCIÓN}

En los últimos años hemos asistido a un cambio radical en el modelo comunicativo. Esta transición no solo ha afectado a los usuarios de internet, que cada vez participan más en el nuevo entorno comunicativo, sino que los propios periodistas han tenido que incluir en sus rutinas diarias otras herramientas nacidas del desarrollo de la web 2.0. Las redes sociales han transformado el ecosistema mediático. La actualidad informativa pasa por un proceso en el cual el usuario es un creador, consumidor y distribuidor de contenidos; en definitiva un "prosumer" 2.

"Las promesas del nuevo entorno mediático suscitan expectativas de un flujo más libre de ideas y contenidos. Inspirados por esos ideales, los consumidores luchan por el derecho a participar más plenamente en su cultura" (Jenkins, 2006, p.28). Una cultura que ya no es propiedad únicamente del periodista que trabaja en un medio y por tanto, obliga a los profesionales de la información a estar más atentos que nunca a los cambios que se están viviendo en un ciberespacio que ya no excluye a nadie; en el que los intercambios de información han pasado de tener un trazado vertical a convertirse en un flujo horizontal de información interconectada.

Las redes sociales empiezan a ocupar un puesto importante en las redacciones periodísticas. Se trata de una nueva forma que tiene el periodista de rastrear cuál es la última hora de determinados temas o tomar el pulso sobre lo qué interesa o no a los ciudadanos. Así, acontecimientos como el terremoto de Lorca, el movimiento del $15 \mathrm{M}$ o el trágico suceso de la desaparición de Marta del Castillo han puesto de relieve la importancia que estas nuevas plataformas sociales han adquirido en el seno de las redacciones periodísticas, donde hoy más que nunca Twitter o Facebook son dos vías más de información para los periodistas del nuevo siglo.

Ante la influencia que los Social Media están provocando en la vida de la gente y en las nuevas formas de trabajo de los periodista, este artículo pretende abrir una reflexión y arrojar algunas claves del papel que están jugando las redes sociales en el trabajo de los periodistas y de qué forma están utilizando ellos estos recursos.

\subsection{Redes sociales, un nuevo objeto de estudio en Comunicación}

Las redes sociales son un fenómeno nacido de las nuevas tecnologías. Nos encontramos ante nuevas formas de comunicación que afectan a la vida de los ciudadanos y a la labor profesional de los periodistas, quienes han encontrado en estas herramientas nuevos recursos informativos para elaborar sus noticias. Por ello, desde una vertiente investigadora es pertinente estudiar y analizar cómo los

\footnotetext{
${ }^{2}$ En el libro de 1980 The Third Wave, el futurólogo Alvin Toffler acuñó el término "prosumidor" cuando hizo predicciones sobre los roles de los productores y los consumidores, aunque ya se había referido al tema desde 1970 en su libro Future Shock
} 
profesionales de la información han adaptado los Social Media a su labor diaria y cómo aprovechan las posibilidades que ofrecen estas nuevas plataformas comunicativas que, cada vez más, repercuten en su quehacer diario.

El presente artículo aborda el comienzo de una investigación que desde la comunicación se está realizando con respecto a la praxis periodística ante el avance de los nuevos medios.

En un contexto donde la multiplicidad de fuentes y la superabundancia de información inundan las redacciones periodísticas, se hace necesario averiguar cuáles son los usos que los periodistas están dando a las nuevas herramientas 2.0 e intentar dilucidar cuáles son las claves para que los profesionales de la información obtengan un resultado óptimo, que se traduzca en una mayor calidad periodística y repercutan en el bienestar de la sociedad. Si tenemos en cuenta que el derecho a la información es uno de los principios básicos de nuestra constitución, cualquier mejora que se haga en pro de la calidad periodística repercutirá directamente en los contenidos que llegan al ciudadano, clave fundamental de la cadena informativa.

Hasta ahora la mayor parte de artículos y estudios sobre el tema se han detenido en una fase de meta información sobre las redes sociales. Además, las exploraciones se han hecho en gran medida desde el área de marketing o publicidad, generando teorías cuyo único interés ha sido el del mercado. Sin embargo, existe una escasez de estudios desde el punto de vista periodístico sobre el valor y la utilidad de estas nuevas plataformas comunicativas.

Por ello, es interesante superar esa fase descriptiva y analizar un aspecto más utilitario de estas redes dentro de un entorno periodístico real. "Adentrarse con naturalidad y explotar al máximo las posibilidades periodísticas de las redes sociales es aún una tarea pendiente, algo por hacer en el ciberperiodismo" (Noguera, 2009, p. 178). Se trata por tanto de un proceso clave, si se tienen en cuenta no solo las necesidades de los periodistas, sino también las de los estudiantes de comunicación que como nativos digitales utilizan ya estas herramientas de forma naturalizada.

\subsection{El nuevo entorno 2.0}

En un escenario donde el $83 \%$ de los internautas españoles utilizan alguna red social, el $40 \%$ la visita más de una vez al día y el $20 \%$ forma parte de al menos tres redes sociales (Fundación Orange, 2011), se hace necesario estudiar las potencialidades que éstas ofrecen a los periodistas, quienes sin duda han encontrado en ellas "fuentes" informativas que les ayudan a buscar pistas y pulsar la realidad de una manera más rápida, aunque no más segura. Por ello, el periodista debe ser capaz de filtrar, comprobar y acreditar esos nuevos contenidos que se generan en los Social Media y llevar a cabo un trabajo informativo completo y eficaz, sin olvidar la veracidad y el rigor que debe desprenderse de cualquier noticia. 
En los últimos tiempos, asistimos a un cambio en el tradicional paradigma de hacer periodismo. Antes el periodista salía a la calle a localizar sus fuentes y a buscar aquella información que desde la mesa de la redacción no podía encontrar. Hoy, esto ha cambiado. Internet, y últimamente, las redes sociales han hecho que los profesionales de la información recurran, cada vez más, a este tipo de plataformas digitales para buscar informaciones, testimonios o datos con los que poder construir sus noticias. La dificultad surge cuando, ante la incertidumbre del nuevo entorno digital, el periodista ignora cómo tratar y usar plataformas sociales cayendo en una deficitaria utilización y un escaso tratamiento informativo del fenómeno.

Las redes sociales han conmocionado los pilares clásicos en los que se sustentaban los tradicionales sistemas y modelos de comunicación mediática. Por primera vez, en la historia de los Mass Media, el público tradicional, denominado "pasivo" por las clásicas teorías de comunicación (Wolf, 1987), puede interactuar con los medios y es cada vez "más activo para resistir el flujo de información que se le dirige" (Wolton, 2010, p.22). Pasando de una estructura vertical y unidireccional a un sistema horizontal, bidireccional e interactivo. Mientras antes el lector consumía los textos sin mucha capacidad de acción, ahora sus comentarios, búsquedas o publicaciones pasan a formar parte del contenido mediático en numerosas ocasiones.

El tradicional modelo periodístico que todos conocemos se ha visto superado por un sistema en donde las relaciones entre fuente, periodista y receptor se han transformado completamente. Las fuentes se han convertido en medios y los receptores son también fuentes que, a través de las redes sociales o sus espacios personales en la Web, pueden nutrir de información al periodista que está en la redacción elaborando una noticia. Cualquiera puede publicar, o incluso leer un dato y rectificarlo inmediatamente con las múltiples herramientas de participación que ofrecen los nuevos medios. Se trata de espacios, hasta ahora exclusivos de los periodistas, en donde el User Generated Content (CGU), término sobre el que se viene investigando desde los años 90, (García De Torres, 2010) ha encontrado su lugar.

Las nuevas tecnologías permiten a los periodistas acceder a multitud de fuentes, documentos y archivos en un tiempo record. Internet es una fuente permanente de consulta para los profesionales que cada vez invierten más tiempo en navegar por la Red. How Spanish Journalists Are Using Twitter, el último estudio publicado por Labápart, indica que más del $90 \%$ de los periodistas encuestados consideran Twitter como la mejor red social para buscar información y contactar con fuentes. Así mismo, este estudio pone de manifiesto que los periodistas utilizan las redes sociales, en gran medida, para publicar y distribuir información (Carrera, 2011).

A pesar de la rapidez e inmediatez que conllevan los medios digitales, los profesionales consideran que es necesario contrastarlo siempre, pero las pruebas evidencian que esto no es así habitualmente. Fácilmente se encuentran noticias en diarios digitales sin fuentes informativas o como máximo con una fuente. Un ejemplo de esta aseveración lo encontramos en el análisis llevado a cabo en un diario digital de Madrid que reflejaba que la media de fuentes encontradas en las noticias 
codificadas durante una semana era de 0,88 lo que suponía que en algunas informaciones no existía atribución alguna a una fuente informativa; es decir, "no sé conocía de dónde salía la información" (Herrero, 2010, p. 152).

\subsection{Las redes sociales en el periodismo}

\subsubsection{Usuarios, contenidos y fuentes periodísticas}

Hoy en día la audiencia puede interactuar con los nuevos medios y el rastro que va dejando en la web a través de sus comentarios, búsquedas o publicaciones pasa a formar parte del contenido mediático. Dejando atrás el clásico, aunque sin perderlo de vista, modelo unidireccional de comunicación ya no se puede hablar de una jerarquía piramidal donde estaba claro el papel que jugaban receptor y emisor en el proceso comunicacional. Una dinámica mediática que ya avanzaba Walter Benjamín en su "Obra de arte en la época de su reproductibilidad técnica" cuando afirmaba: "La distinción entre autor y lector está perdiendo su dimensión fundamental para convertirse en una diferencia funcional ligada a las circunstancias: el lector está siempre dispuesto a ser escritor" (2010, p. 37).

Sin duda, una afirmación que encuentra hoy su máximo exponente en los blogueros, tuiteros y demás perfiles que en los últimos años han inundado la web de contenidos, en algunos casos relevantes; pero en su mayoría, insustanciales y superficiales. A. Moles también se refería a un fenómeno parecido cuando hablaba de los medios de comunicación consideraba que existía un "problema de opulencia comunicativa" (Moles, 1971, citado por Carrera, 2008) que con la llegada de Internet se ha visto acelerado. Parece que bajo la apariencia liberadora y creadora de la Web se esconden numerosas opiniones cuya finalidad es que sean vistas por otros y encontrar la ansiada "identidad digital" de éste nuevo siglo sin necesidad de atender completamente a los contenidos. Todos opinan, todos quieren tener su espacio en la Web, pero no siempre se aportan informaciones sustanciales o que realmente puedan interesar al público.

El usuario de Internet es un sujeto que ha pasado de recibir información a crearla y publicarla sin necesidad de ser periodista. Esta reconversión ha hecho que un público que consumía noticias de forma masiva, lo haga de una forma más selectiva y contando con una dosis de autonomía que le permite valorar, difundir o interaccionar con la propia información.

La posibilidad de interactuar y emitir, por parte de los tradicionales consumidores pasivos de información, ha llevado a algunos autores a declarar en bancarrota el concepto de masa, sustituyéndolo por el de un usuario autónomo capaz de generar información y difundirla públicamente a través de la web. "En la actualidad son muchas las voces que declaran los conceptos de "masa" y de "culturas de masas" teóricamente agotados, (...) sobre todo tras el advenimiento de medios interactivos como Internet". (Carrera, 2008, p.128). 
Sin embargo, la definición que McLuhan avanzó en 1977 sobre la "masa" si se podría volver a recuperar para hablar de Internet ya que entiende el fenómeno de masa no como una cuestión física sino de velocidad "un fenómeno de velocidad eléctrica, no de cantidad fisica". A mayor "enganche" con el medio y mayor implicación con más gente más masa hay, al entender de McLuhan.

Cuando habla de "medios de comunicación de masas" no está hablando tanto del número de personas al que se dirige sino más bien que todo el mundo está implicado en ellos al mismo tiempo, como él bien lo define la "producción en masa" se convierte en "un abrazo inclusivo" (McLuhan, 2009, p.396). Se están difuminando, cada vez más, las fronteras entre los diferentes medios de comunicación tanto tradicionales como nuevos. Un proceso en el que como defiende Castells, se está pasando de una comunicación de masas a la "autocomunicación de masas" (2009, p.92).

De esta forma Internet, y las redes sociales en particular, cada vez enganchan a más gente, convirtiéndose en la actualidad en los medios de masas por excelencia.

Es en este panorama informativo, y mediático, en el que el concepto de usuario pasivo ha dejado paso al User Generated Content, algo que por otro lado, demandan las empresas de comunicación. Cada vez más, los propios medios invitan a sus audiencias a participar en la generación de contenidos (Quinn \& Lamble, 2008). Nunca ningún otro medio ha formado parte de la vida de la gente de forma tan usual como Internet, y todas las nuevas formas de comunicación que surgen en su seno. En palabras de la profesora Pilar Carrera, estaríamos ante una "traslación de la comunicación intrapersonal de lo Off Line a lo On Line", con toda una serie de condicionantes que hacen que la comunicación en la red sea distinta a la interpersonal, pero la conversación generada por los media, que antes se producía offline (en la cafetería, con los amigos...) ahora en parte se ha trasladado a lo online.

La cuestión, que subyace en este debate, es que el término masa ha sido sustituido por el término usuario dotando a éste de una autonomía de la que la masa carecía. El nuevo medio, como los demás medios de comunicación, se dirigen a un importante número de personas, pero en esta ocasión Internet ha hecho que los contenidos que existen allí sean universalmente accesibles.

La automatización no afecta solamente a la producción, sino a todas las fases del consumo y comercialización; en un circuito automatizado, el consumidor se convierte en productor, del mismo modo que el lector del mosaico de la prensa telegráfica se hace sus propias noticias o, simplemente, en sus propias noticias" (McLuhan, 2009, p. 396).

Como se ha visto anteriormente, con la llegada de Internet los límites entre fuente, emisor y receptor son cada vez más difusos. Esta situación lleva a que cualquier usuario pueda acudir directamente a la fuente sin necesidad de pasar antes por el 
medio o que un periodista pueda hacerse eco de una información que alguien ha publicado en su blog, o su website, sin someterla a los filtros habituales de comprobación. Este panorama lleva por tanto a una pérdida de control y comprobación de ciertas informaciones publicadas por diversas fuentes.

En el periodismo digital existe una tendencia generalizada a no citar las fuentes, el periodista se apropia así de una información que alguien le ha facilitado. En la mayor parte de las ocasiones las informaciones provienen de agencias llegando a convertirse en el recurso básico de "los periódicos on-line en el 47, 35\% de los casos" (Diezhandino \& Carrera, 2008, pág.68-69). Un fenómeno que nos hace cuestionarnos quién es el que hoy en día hace realmente periodismo: ¿La agencia o el medio de comunicación?

Las nuevas tecnologías están permitiendo a los periodistas acceder a multitud de fuentes, documentos y archivos en un tiempo record. Internet es una fuente permanente de consulta para los profesionales que cada vez invierten más tiempo en navegar por la Red o consultar informaciones. A pesar de esta realidad, su confianza en las fuentes es menor que en la que tienen por las fuentes tradicionales. A pesar de la rapidez e inmediatez que conllevan los medios digitales, los profesionales consideran que es necesario contrastarlo siempre, pero las pruebas evidencian que esto no es así habitualmente.

\subsection{El surgimiento de libros de estilo en las redacciones}

Los manuales de estilo, en el ámbito periodístico, son un conjunto de normas específicas para redactar, diseñar o utilizar de la manera más correcta posible el idioma en el que nos vamos a comunicar. Se trata de una serie de pautas que cumplen perfectamente la función de usabilidad y permiten al periodista trabajar dentro de un marco de referencia a la hora de redactar noticias y solventar posibles dudas. Algunos de estos manuales contemplan también la dimensión ética de la profesión, ya que "a los editores les inquieta mucho que los periodistas puedan dañar la credibilidad, la imagen y la reputación de la empresa" (Herrera, 2011, p.15)

Aunque, responsables de la estrategia digital de algunos medios españoles como 20Minutos.es o Unidad Editorial ${ }^{3}$ coinciden en que no existen normas escritas sobre cómo deben utilizar los periodistas las redes sociales, parece que si están apareciendo de forma discreta los primeros borradores sobre cómo trabajar con los Social Media en el entorno periodístico. De hecho, el 33\% de los periodistas entrevistados en el estudio How Spanish Journalists Are Using Twitter realizado por Labápart afirman que su medio está trabajando en la elaboración de este tipo de manuales. Pero, más de un $50 \%$ de los encuestados aseguran no tener una guía de uso para el manejo de las redes sociales.

\footnotetext{
${ }^{3}$ Esta información se ha extraído de algunas entrevistas informales que se han mantenido con algunos de los responsables de la estrategia digital de diferentes medios de comunicación
} 
En España existe el caso reciente de la Agencia EFE que en marzo de 2011 lanzó el blog: Estilo. Manual para nuevos medios, una iniciativa con la que se pretende dar respuestas a las dudas más comunes que surgen en relación con el uso de Internet en general y de los nuevos medios en particular. La estructura de este manual responde en esencia a la "inteligencia colectiva" de la que habló Piérre Levy en 1997, y que otros muchos autores como Jenkins han recuperado para señalar la importancia de construir contenidos en Internet a partir del conocimiento de las diferentes comunidades. Se trata de un manual en el que de una forma interactiva, abierta y colaborativa se generan contenidos, instrucciones y normas para poder comunicar y manejarse fácilmente en los nuevos entornos digitales.

En Europa, la agencia France Press ha prohibido a sus periodistas que utilicen Facebook como fuente de información exclusiva, sin contrastar con otras. Esta medida se ha tomado después de que algunos medios publicaran fotos de Benazir Bhutto, recogidas en Facebook, que eran falsas. Por su parte, Reuters ha creado una guía de estilo en la que da una serie de indicaciones a sus periodistas sobre cómo utilizar las redes sociales para elaborar informaciones y de qué forma verificar y contrastar lo que encuentran en ellas.

The Washington Post es otro de los diarios que ha elaborado una guía sobre cómo deben trabajar sus periodistas en las redes sociales. La guía se basa en tres puntos claves: "cómo debe utilizar la redacción en Facebook, Twitter y otras redes sociales", "utilizar las redes sociales como herramientas para informar" y "emplear las herramientas de las redes sociales por razones personales". El periódico admite en esta guía que las redes sociales son un medio de comunicación más y que, dado su uso cotidiano en el día a día, los periodistas deben aprender a usarlas como una herramienta valiosa para la recopilación y difusión de noticias. "Los periodistas del Washington Post tienen que saber que al usar este tipo de recursos lo están haciendo como profesionales del periódico ${ }^{\prime \prime}$, afirmaba en 2009 el defensor del lector de esta cabecera en su blog.

En conclusión, es evidente que el periodista ya no puede vivir al margen de esta nueva realidad que le brindan las redes sociales por lo tanto es necesario establecer unos nuevos códigos periodísticos que sirvan al periodista de guía para poder trabajar con estas nuevas herramientas sin deteriorar, por ello, las exigencias de contrastación y comprobación que siempre han definido al buen periodismo. Y es que "las tecnologías han variado, pero los principios que subyacen en ellas están aún vigentes" (Bill Kovach \& Tom Rosenstiel, 2003, pág. 35).

En los últimos años, los medios de comunicación españoles han entrado en una carrera por estar presentes en las plataformas sociales. Esta carrera, al igual que

\footnotetext{
${ }^{4}$ Estas declaraciones están recogidas en el blog de Andrew Alexander, defensor del lector en el Washington Post. Disponible en:

http://voices.washingtonpost.com/ombudsmanblog/2009/09/post_editor_ends_tweets_as_new.html ?wprss $=$ ombudsman- $\underline{\text { blog }}$
} 
ocurrió con la aparición de los primeros periódicos on line, comenzó de una manera muy básica. Si los primeros periódicos digitales no eran más que el volcado del contenido de los tradicionales, la presencia inicial de los medios en las redes sociales consistía en agregar un botón que permitiera compartir una determinada noticia en una red social. Esta realidad, que no queda tan lejos, ha evolucionado hasta la creación de verdaderas comunidades de participación ciudadana y perfiles de medios de comunicación en las propias plataformas sociales para llevar a cabo eficientes estrategias de fidelización de audiencias.

Tras un breve análisis de los principales medios de comunicación se presenta a continuación una pequeña radiografía del estado que atraviesan los principales diarios de referencia en España en el ámbito de los Social Media y la participación. La siguiente descripción agrupa las principales innovaciones que estos diarios han llevado a cabo en los últimos meses.

\subsection{Puesta en marcha de algunas estrategias digitales en los medios}

\subsubsection{Dentro de España}

Una de las últimas aportaciones se ha podido encontrar en el diario El País que ha creado la sección de política con la que se pretende fomentar la participación de los lectores en un tema que ahora mismo consideran prioritario. "La tecnología permite, tanto a medios tradicionales como a ciudadanos, una implicación mayor en la acción política, fundamentalmente a través de internet y con las redes sociales como máximo exponente". (El País.com, 2011)

A través de Eskup, la red social que este mismo diario lanzo en Junio de 2010, los usuarios pueden opinar, participar en las conversaciones que se generen en esta nueva sección y seguir a los periodistas especializados en esta área, que darán su opinión y analizarán lo que está ocurriendo en la actualidad política. Pretenden utilizar lo que ya tenían con Eskup para generar conversaciones e intercambios sobre un tema más especializado como es la política y que ellos consideran de interés público.

Otra de las aportaciones más relevantes en los últimos meses ha sido la pizarra digital de 20 Minutos, una iniciativa con la que los responsables del diario gratuito pretenden acercar a sus lectores los temas sobre los que están trabajando en el día e implicarles pidiéndoles su opinión y sugerencias con respecto a otros temas sobre los que poder trabajar.

En cuanto a otros diarios como ABC, La Vanguardia o El Mundo no se han encontrado grandes diferencias con respecto a los anteriores. Todos ellos están presentes en las redes sociales, en sus noticias dan la oportunidad a sus usuarios de compartir las informaciones en su perfil y todos ellos tienen su propia página en estas plataformas. 


\subsubsection{Fuera de España}

Fuera de nuestras fronteras, algunos medios de comunicación han comenzado una verdadera carrera de fondo por encontrar un lugar en las redes sociales.

Este es el caso del diario británico The Guardian que en los últimos meses ha apostado por diversificar sus temáticas en las redes sociales. De esta manera, ha creado en Twitter 50 perfiles y en Facebook cuenta con 30. Al igual que ocurre en los diarios digitales españoles, el responsable de Desarrollo de Medios Sociales de Guardian News Media, Meg Pickard ${ }^{5}$, ha señalado que en el diario británico no existe un equipo de periodistas dedicados exclusivamente a las redes sociales, sino que la gestión de estos canales de información forma parte de la rutina de estos profesionales.

Las principales funciones que los periodistas de este diario desempeñan en las redes sociales son diferentes según se hable de Twitter o Facebook. Así, mientras en Twitter son referentes y prescriptores, interviniendo también en las conversaciones con sus lectores, en Facebook buscan más publicar aquella información más susceptible de ser compartida por los usuarios. De nuevo, se puede observar como las estrategias periodísticas varían según el canal, no es lo mismo Twitter que Facebook, sin embargo parece claro que es necesario estar en ambas redes si se quiere realmente llevar a cabo un periodismo 2.0 y formar parte de un entornos donde el lector cobra más protagonismo.

Otro caso, se encuentra en la cadena estadounidense de radio y televisión CBS (Columbia Broadcasting System). Este medio de comunicación ha decidido integrar dentro de su parrilla televisiva un programa basado en los temas más comentados dentro de los Social Media, los famosos trending topic o temas del momento. Para ello, la cadena ha apostado por What's Trending un programa televisivo semanal en el que las redes sociales no se utilizan como una herramienta para aportar contenido al programa, sino que el propio contenido que encontramos en las redes sociales es la temática sobre la que gira todo el programa.

Es decir, la conversación social mantenida en estas plataformas se convierte en contenido informativo. Un fenómeno conversacional del que a comienzos del siglo XX ya hablaba el sociólogo francés, Gabriel Tarde, cuando la conversación pasa a formar parte del medio, en el caso de este magacín, a través de plataformas como las redes sociales convirtiendo al medio en un "híbrido" que recupera y aumenta las dinámicas de las relaciones interpersonales.

\footnotetext{
${ }^{5}$ Esta ha sido una de las consideraciones que la Responsable de Desarrollo de Medios Sociales de Guardian News Media, Meg Pickard, hizo en la séptima jornada del 10 en Comunicación sobre gestión de la identidad digital corporativa de ESCACC. Disponible en:

http://www.escacc.cat/escacc/ca/projectes/10_en_comunicacio.html
} 


\section{METODOLOGÍA}

En este artículo se han explorado, en líneas generales, cuáles son las principales tendencias actuales que los medios de comunicación y el periodismo están adoptando ante las redes sociales y su integración en las rutinas periodísticas Tras una revisión de la literatura más actual sobre redes sociales y periodismo, se ha intentado encuadrar el tema teóricamente para pasar a continuación a la parte cuantitativa del estudio. Para ello, se ha elaborado un cuestionario on line integrado por 14 ítems constituidos por preguntas cerradas que combinan preguntas de una sola respuesta y cuestiones de opción múltiple. La muestra elegida para este artículo ha sido de 67 periodistas españoles ${ }^{6}$ que trabajan en medios de comunicación, agencias, empresas privada, como freelance o que no tienen trabajo.

El cuestionario alojado en una plataforma on $\operatorname{line}^{7}$ se ha hecho accesible a los periodistas españoles a través de diferentes canales de difusión. (La web de la FAPE, Twitter, Facebook, Google+ y Linkedim). En este artículo se presentan algunos de los resultados que se han obtenido como parte de un proyecto de investigación más amplio que todavía sigue en curso y del que no se conocen datos definitivos. La siguiente discusión de resultados permite extraer algunas conclusiones y anticipar lo que previsiblemente serán las tendencias actuales en el mundo del periodismo y las redes sociales en España.

\section{ANÁLISIS Y DISCUSIÓN}

En relación, al uso que los periodistas hacen de las redes sociales en su trabajo, el $62 \%$ de los encuestados afirma usar "siempre" las redes sociales en su profesión (tabla 1), El 29\% afirma que utiliza las redes sociales "a veces" y entre un 4 y un 5\% afirman utilizar los Social Media poco o nada, respectivamente. Sin detenernos, por no tener respuestas suficientes, en la naturaleza de los puestos de trabajo que desempeñan los periodistas que han respondido; ya que seguramente no es igual el uso que hace un periodista desde un gabinete de prensa que desde un diario digital si parece existir un uso extendido de estas nuevas plataformas sociales como una herramienta más en el periodismo.

\footnotetext{
${ }^{6}$ Los resultados que se exponen están actualizados a día 24 de agosto de 2011. La encuesta fue abierta el 27 de julio de 2011 y se cerrará previsiblemente en diciembre de 2011, fecha en la que se comenzarán a analizar los resultados definitivos

7 La plataforma digital en donde se ha alojado la encuesta es e-encuestas se puede consultar en http://www.e- encuesta.com/index.do?gclid=COjTjebG0awCFQMPfAodqnfy6g
} 


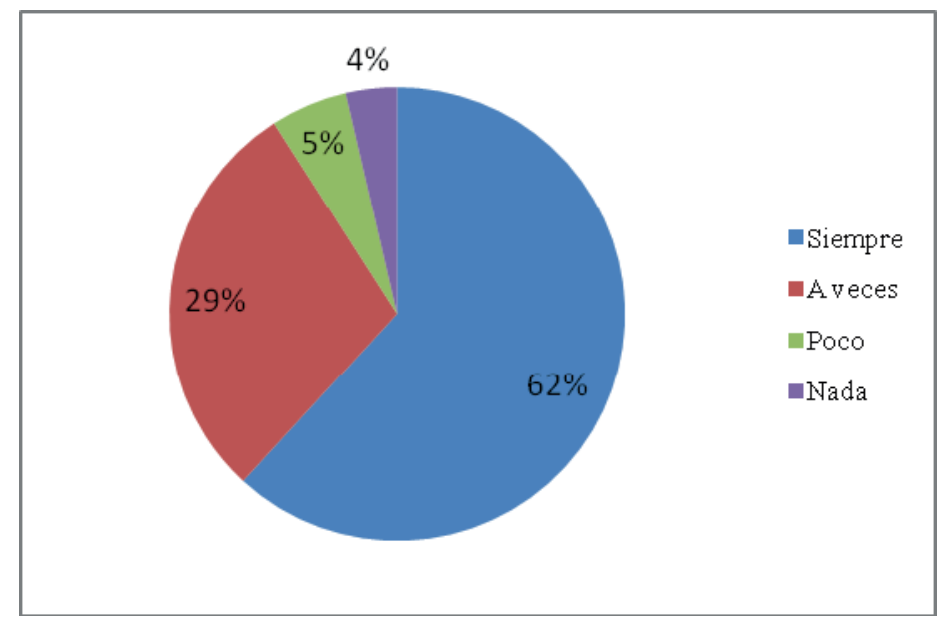

Figura 1. Uso redes sociales trabajo periodistas.

Conocer para qué están utilizando los periodistas estas redes sociales es otra de las claves de esta investigación. Por tanto, una de las preguntas que se consideran esenciales en este trabajo es saber cuál es la aplicación práctica que tiene para los profesionales de la información el uso de estas plataformas.

Un $65,45 \%$ de los periodistas encuestados reconoce usar las redes sociales para difundir información del medio para el que trabaja. Una labor que los responsables de la estrategia digital de los medios fomentan entre sus empleados para tener mayor visibilidad en los Social Media, además, esto sirve probablemente al propio periodista para difundir su trabajo y crearse, poco a poco, una imagen de marca, una idea también muy extendida actualmente en la profesión.

El 63\% de los periodistas encuestados afirman usar las redes sociales para conocer la opinión de la gente (tabla 2). Más de la mitad de los periodistas encuestados, un $56,4 \%$, aseguran utilizar las redes sociales para detectar nuevos temas y para buscar información nueva. Una tendencia que coincide con la opinión de otros autores como la de Ángeles Fernández Barrero que se refiere a las redes sociales como nuevos "yacimientos de material noticioso" (2010, p.2). Sin duda, los periodistas parecen haber encontrado en estas nuevas plataformas una vía para conocer cuáles son los temas que pueden interesar a la audiencia y aquellos asuntos que quizás por no estar contemplados, en las agendas institucionales, se escapan de la cobertura informativa tradicional.

La mitad de los encuestados, un 51\%, afirma utilizar las redes sociales para contactar con fuentes informativas. Por tanto, aunque las fuentes sigan siendo las mismas, probablemente hayan aumentado. La manera de contactar con ellas parece haber cambiado. Ahora la búsqueda de estas fuentes se puede hacer a través de Internet y por tanto, la agenda del periodista se amplía a la misma rapidez que lo hace su presencia en las diferentes redes sociales. Un fenómeno que da mayor acceso a otro tipo de fuentes ciudadanas o institucionales. 
Atraer audiencias (38,2\%) o contrastar informaciones (29\%) son otros de los usos que parecen estar presentes en la actividad social de los periodistas.

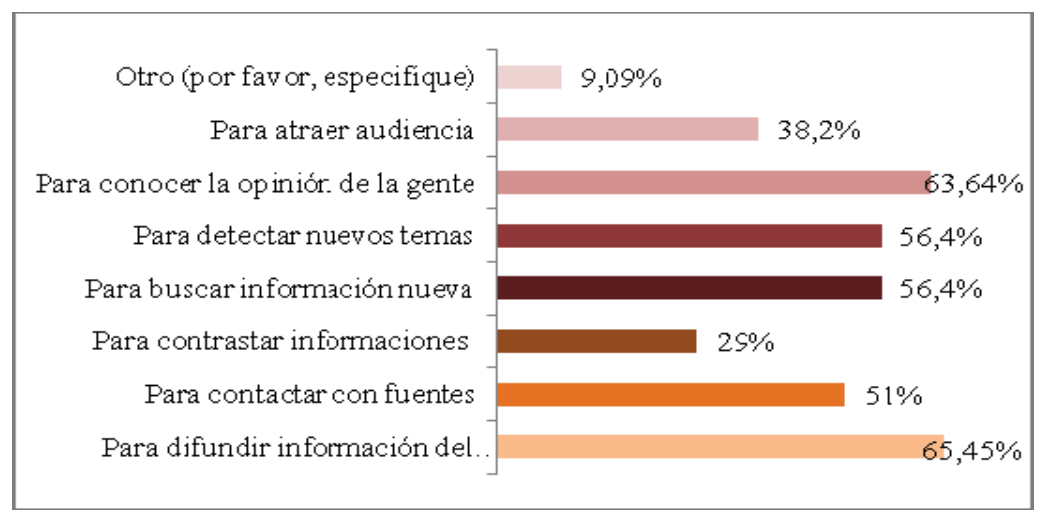

Figura 2. Para que usan los periodistas las redes sociales.

Ante los resultados anteriormente expuestos, se podría pensar que los periodistas confían plenamente en las redes sociales a la hora de abordar su trabajo periodístico. Sin embargo, estas respuestas chocan con el nivel de credibilidad que los informadores dan a los Social Media. Más de la mitad de esos mismos periodistas, un $61 \%$ (Tabla 3) consideran que las informaciones que se encuentran en las redes sociales son menos fiables que las que aparecen en los medios tradicionales. Una opinión que coincide con la presentada en el estudio realizado por la Universidad de George Washington en 2009 y que refleja que el 84\% de los periodistas encuestados considera que la información encontrada en las redes sociales es mucho menos, o levemente menos, fiable que la que se puede encontrar en los medios tradicionales (George Washington University and Cision, 2009, p. 14).

Es decir, mientras que en los últimos años ha aumentado el uso de las redes sociales por parte de los periodistas, el nivel de confianza que estos tienen hacia ellas sigue siendo bajo. Una opinión que desentona con el aumento en el uso que estos profesionales hacen de las plataformas 2.0. Mientras el 62\% de los periodistas reconocen utilizar siempre las redes sociales (tabla 1), sólo un $4 \%$ otorga credibilidad a los contenidos que en ellas se exponen.

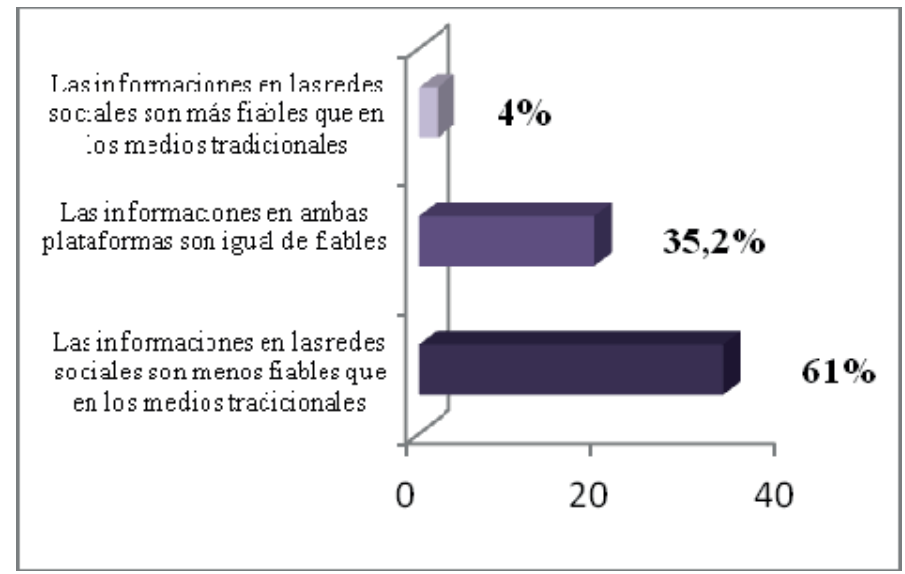

Figura 3. Credibilidad. 


\section{CONCLUSIONES}

Los medios de comunicación están llevando a cabo diferentes estrategias para conseguir tener un lugar en las redes sociales, las cuales se están posicionando como un nuevo modelo de negocio que es necesario explotar. Estas estrategias van desde crear sus propios perfiles para estar presentes en las múltiples plataformas hasta diseñar guías de estilo o recomendaciones para que sus periodistas usen de forma diligente los Social Media.

En esta carrera por estar en la web 2.0, los medios extranjeros han avanzado más que los medios españoles, donde la incertidumbre y el temor a lo desconocido sigue imperando en las cúpulas directivas de los medios de comunicación.

Abrir un debate en el seno de las redacciones, o entre los profesionales de la información, sobre cómo utilizar y a provechar al máximo las potencialidades que las nuevas plataformas sociales le ofrecen es clave para el oficio. Este debate debe partir entre otras esferas del mundo académico, donde las redes sociales han pasado a formar parte de la agenda investigadora en medios de comunicación.

A la vista de los resultados se puede considerar que a día de hoy los periodistas españoles están utilizando las redes sociales en su trabajo diario para contactar con fuentes, difundir/distribuir información se su medio o de otros medios y para detectar nuevos temas. Aunque, en la mayoría de los profesionales sigue existiendo una desconfianza a estas nuevas herramientas y no le otorgan la misma credibilidad que si ocurre con otros medios tradicionales de comunicación.

\section{REFERENCIAS}

Bowman, S.;\& Willis, C. (2003). Nosotros, el medio. Cómo las audiencias están modelando el futuro de las noticias y la información. The Media Center at The American Press Institute. Reston (Online Journalism Review.). Colombia: El Tiempo. Recuperado el 5 de junio de 2011, de

http://www.hypergene.net/wemedia/download/we_media_espanol.pdf

Carrera, P. (2011).Join the conversation: How spanish journalists are using social media. Recuperado el 1 de Agosto de 2011, de

http://www.labapart.org/breaking_news.html.

Edo, C. (2003). Periodismo informativo e interpretativo: el impacto de Internet en la noticia, las fuentes y los géneros ( $1^{\mathrm{a}}$ ed.). Comunicacion Socia. Sevilla: Ediciones y Publicaciones.

Fernández, F. (2011). Making-Journal, una oportunidad para el periodismo en la Red. En Flores, J. M. Reinventar el periodismo y los medios: apuntes sobre el estado del arte en la construccion del ciberperiodismo (pp. 71-78). Madrid: Fragua. 
Fundación Orange (2011). España. Informe anual sobre el desarrollo de la sociedad de la información. Recuperado el 30 de Mayo de 2011, de http://www.informeeespana.es/docs/eE2011.pdf.

García de Torres, E. (2010). Contenido generado por el usuario: aproximación al estado de la cuestión. El Profesional de la Información, 19(6): 585-594.

George Washington University \& Cision (2009). 2009 Social Media E Online Usage Study. Washington. Recuperado el 5 de julio de 2011, de http://www.gwu.edu/ newsctr/10/pdfs/gw_cision_sm_study_09.PDF.

Henriksson, T.(2011). CBS's new social media-powered TV show goes on air. Recuperado el 25 de agosto de 2011, de

http://www.editorsweblog.org/web_20/2011/05/cbss_new_social_mediapowered_tv_show_go.php.

Herrera, S. (2011). Social media policies en los medios tradicionales, cuando el sentido común no es suficiente. En Larrondo, A. \& Meldo, K.: La transformación del espacio mediático (pp. 418-436). Bilbao: Universidad del País Vasco.

Herrero, E. (2010). El tratamiento informativo del suceso en el periódico digital Madrid. En VVAA. El periodismo digital desde la perspectiva de la investigación universitaria (pp.145-156). Huesca: Asociación de la Prensa de Aragón..

Jenkins, H. (2006). Convergence Culture: Where Old and New Media Collide. New York: NYU Press.

Kovach, B. \& Rosenstiel, T. (2003). Los elementos del periodismo (1 $1^{\mathrm{a}}$ ed.). Madrid: Aguilar.

Lévy, P. (1997). L'intelligence collective: pour une anthropologie du cyberspace. Paris: La Découverte.

Mcquail, D. (2000). Introduccion a la teoría de la comunicacion de masas ( $3^{\mathrm{a}}$ ed.). Barcelona: Paidos.

Oriella Pr Network (2011). The State of journalism in 2011. Oriella PR Network Digital Journalism Study.

Parra Valcarce, D. (2004). Ciberperiodismo. Madrid: Sintesis.

Scolari, C. (2008). Hipermediaciones: elementos para una teoria de la comunicacion digital interactiva (1 ${ }^{\mathrm{a} e d}$.). Barcelona: Gedisa.

Tarde, G. (1986). La Opinión y la multitud. España: Taurus. 
Wolf, M. (1987). La investigacion de la comunicacion de masas (1 ${ }^{\mathrm{a}}$ ed.). Barcelona: Paidos.

Wolton, D. (2010). Informar no es comunicar: contra la ideologia tecnologica. Barcelona: Gedisa.

\section{Eva Herrero-Curiel}

licenciada en periodismo (UC3M) y en psicología (UAM). En 2009 tuvo una beca de posgrado para cursar el Máster de Investigación Aplicada en Medios de Comunicación en la Universidad Carlos III de Madrid, obtuvo Matrícula de Honor en su Trabajo Fin de Máster. En la actualidad, trabaja en el Departamento de Periodismo y Comunicación Audiovisual de la Facultad de Humanidades, Comunicación y Documentación de la Universidad Carlos III de Madrid 